\title{
Event-by-event fluctuations and the QGP phase transition
}

\author{
Tapan K. Nayak $\ddagger$ \\ CERN, CH-1211, Geneva-23, Switzerland \\ E-mail: Tapan.Nayak@cern.ch
}

\begin{abstract}
Fluctuations of thermodynamic quantities are fundamental to the study of QGP phase transition. Event-by-event fluctuations of many quantities have been studied by dedicated heavy-ion experiments. A brief review of recent experimental results is presented. The prospect for future study of fluctuations is discussed.
\end{abstract}

\section{Introduction}

Fluctuations are of fundamental importance for studying perturbation of a thermodynamic system. Several thermodynamic quantities show varying fluctuation patterns when the system undergoes a phase transition. Moreover, fluctuations are sensitive to the nature of the transition. Of particular interest to us is the fluctuation induced by the phase transition between normal hadronic matter and the quark-gluon plasma (QGP), a condition similar to that existed within a few tens of microseconds of the early universe. Event-by-event fluctuations of thermodynamic quantities measured in high energy heavy-ion collisions provide a reasonable framework for studying the nature of the QGP phase transition in the laboratory [1]. Large fluctuations in energy density are expected if the phase transition is of first order whereas a second order phase transition may lead to a divergence in specific heat. An increase in the fluctuation in energy density for a second order transition may be expected due to long range correlations in the system. Furthermore, the prospect of locating the critical point of the QGP phase transition where the fluctuations are predicted to be largely enhanced [2] makes this study rather interesting and challenging.

The rapid development in the field of event-by-event fluctuations in recent years is

related to the availability of large amount of high multiplicity data from dedicated heavyion experiments at the CERN-SPS and BNL-RHIC. Although tremendous progress has been made in our understanding of the fluctuations, still the promise of the fluctuation measures to provide a clear understanding of the QGP phase transition has yet to be realized. Recent advances in theoretical aspects, especially from lattice computations, and plans for dedicated experiments give much needed assurance. There are several $\ddagger$ On leave from Variable Energy Cyclotron Centre, Kolkata, India 
effects which make the study of fluctuations non-trivial. One of the most important issues concerns the separation of statistical and known physics contributions from the measured fluctuations in order to identify the dynamical part associated with the phase transition.

In case of high energy heavy-ion collisions, there are several sources which contribute to the measured fluctuation, which include: (a) geometrical (impact parameter, number of participants, detector acceptance), (b) energy, momentum and charge conservation, (c) anisotropic flow, (d) Bose-Einstein correlations, (d) resonance decays, (e) contribution from jets and mini-jets, etc. These effects must be taken into account in order to infer about the fluctuation from dynamical origin related to that of the phase transition. Present analyses use one or more of the following methods for this purpose:

- construction of mixed events which take care of instrumental effects,

- simulation of fluctuations originating from statistical and known physics sources,

- construction of fluctuation measures which are robust against known fluctuations.

We give a brief review of some of the fluctuation measures which have been studied in heavy-ion collisions. These include fluctuations in multiplicity, particle ratio, net charge, mean transverse momentum $\left\langle p_{\mathrm{T}}\right\rangle$, and methods of balance functions and long range correlations. Finally we give an outlook on the near term plans including the prospect of studying event-by-event physics in the ALICE experiment at the LHC.

\section{Multiplicity fluctuations}

Multiplicity of produced particles characterizes the evolving system in a heavy-ion collision and thus fluctuation in multiplicity may provide a distinct signal of the QGP phase transition [1, 3. Since multiplicity distributions for narrow centrality bins can be described by Gaussian distributions, their fluctuations are expressed in terms of scaled variance, defined as, $\omega=\operatorname{var}(N) /\langle N\rangle$, where $\langle N\rangle$ and $\operatorname{var}(N)$ represent the variance and mean of the multiplicity distribution, respectively. Figure 1 shows observed scaled variance for SPS and RHIC energies. The results from the WA98 experiment [3], those corresponding to photons and charged particles, are compared to different model calculations. Although the experimental data is consistent with the model calculations within the quoted error bars, the increasing trend of fluctuation for charged particles towards peripheral collisions is clearly visible. The scaled variance of charged particles as a function of centrality from NA49 [4] shows a non-monotonic behaviour. The PHENIX

data [5] for $\mathrm{Cu}-\mathrm{Cu}$ collisions at $\sqrt{s_{\mathrm{NN}}}=62.4 \mathrm{GeV}$ shows a small structure for non-central collisions whereas at higher energies the data are smoother. Detailed understanding of these results would require considerations of centrality selection and detector effects.

\section{Net charge fluctuations}

Fluctuations of conserved quantities like electric charge, baryon number or strangeness are predicted to be significantly reduced in a QGP scenario as they are generated in the 

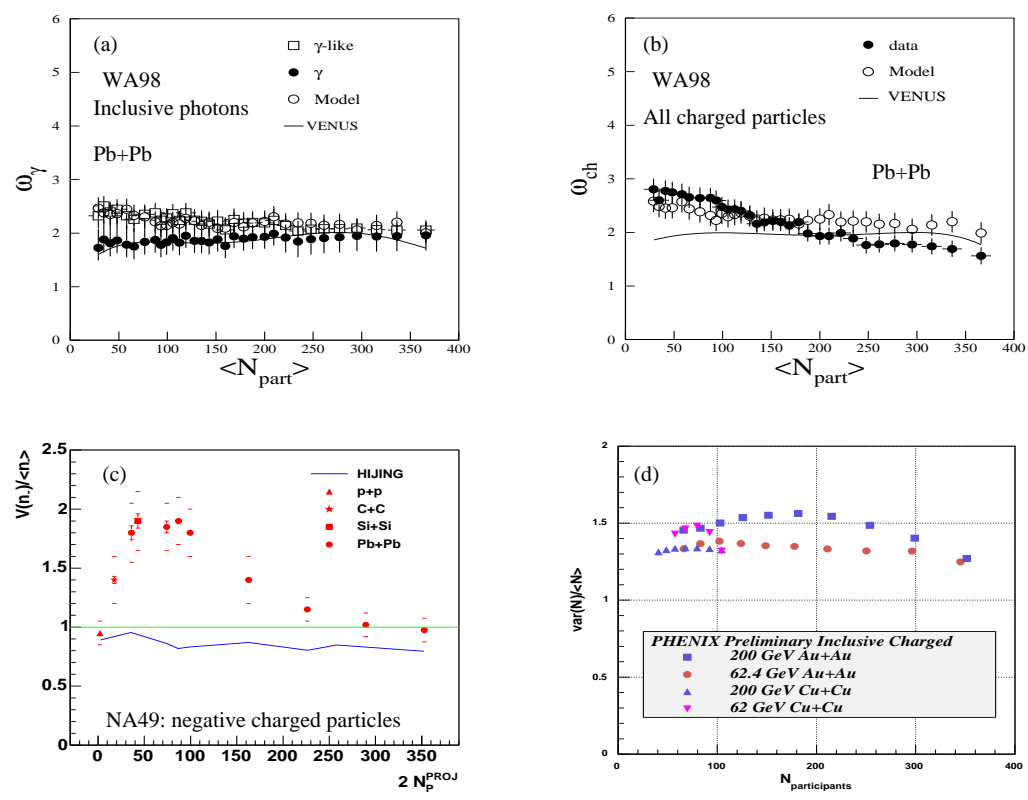

Figure 1. Multiplicity fluctuations at SPS and RHIC energies.

early plasma stage of the system created in heavy-ion collisions with quark and gluon degrees of freedom [6, 7]. The fluctuation generated at the QGP stage will increase as the system evolves in time [8, 9]. Net charge fluctuations have been measured by experiments at SPS and RHIC using different fluctuation measures. Among these are $\Phi_{q}$ of NA49 [10], $\nu_{+-, d y n}$ of STAR [11] and $v(Q)$ as well as $\nu_{+-, d y n}$ used by PHENIX [12]. A common framework which relates these variables [13 has been used to compile the available results [14]. This is shown in Fig. 2, along with predictions from independent particle emission, quark coalescence, resonance gas and a QGP scenario. Both NA49 and PHENIX results are consistent with the independent particle emission scenario, whereas the result for STAR is close to the case of the quark coalescence model.

Recently, lattice computations [15, 16] have been performed to study hadronic fluctuations. These calculations predict an enhancement of fluctuation in the hadronic phase and suppression of fluctuations in the high temperature phase of the QGP. More interestingly, prominent structure in the higher order moments of net charge distributions have been observed for temperatures close to the transition temperature. The higher moments of net charge distributions can be studied from experimental data. Figure 3 shows the net charge distributions of particles with $p_{\mathrm{T}}$ below $1 \mathrm{GeV} / \mathrm{c}$ for Au$\mathrm{Au}$ collisions at $\sqrt{s_{\mathrm{NN}}}=200 \mathrm{GeV}$ for different centralities in the STAR experiment within a pseudorapidity coverage of $-1 \leq \eta \leq 1$. Efforts are underway to study higher order moments of these distributions by making smaller bins in detector acceptances and $p_{\mathrm{T}}$. 

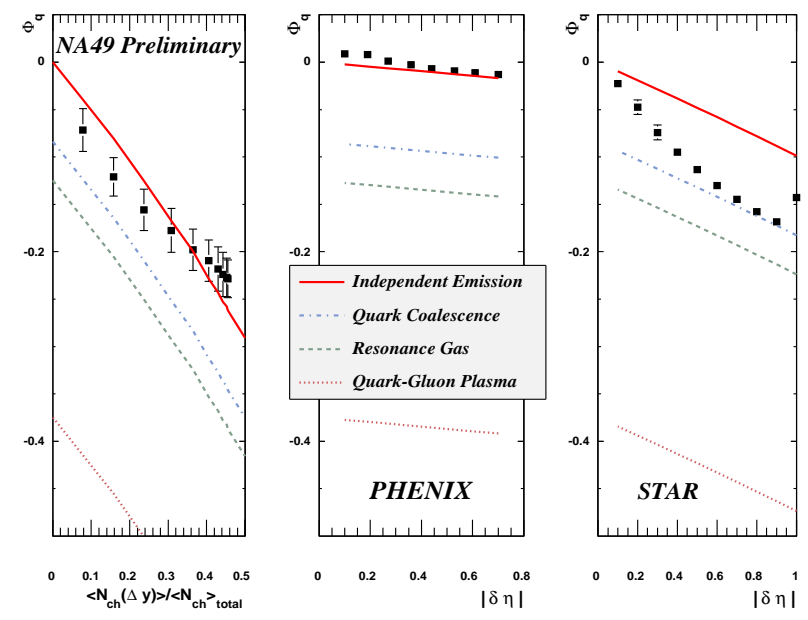

Figure 2. Dynamical fluctuation of net charge for NA49, PHENIX and STAR experiments [14.

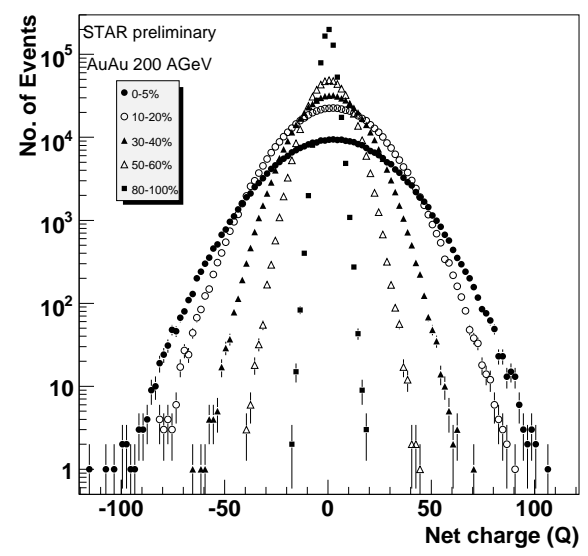

Figure 3. Net charge distributions of particles with $p_{\mathrm{t}}$ below $1 \mathrm{GeV} / \mathrm{c}$ for $\mathrm{Au}-\mathrm{Au}$ collisions at $\sqrt{s_{\mathrm{NN}}}=200 \mathrm{GeV}$ at different centralities.

\section{Fluctuations of particle ratio}

Relative production of different types of particles produced in the hot and dense matter might be affected once the system goes through a phase transition. Of particular interest is the strangeness fluctuation in terms of the ratio of kaons to pions. Large broadening in the yields of kaons to pions has long been predicted because of the differences in free enthalpy of the hadronic and QGP phase. This could be probed through the fluctuation in the $K / \pi$ ratio.

A detailed study at SPS has been carried out at several beam energies [17]. The ratio of inclusive mid-rapidity yields of $\left.\left\langle K^{-}\right\rangle /\left\langle\pi^{-}\right\rangle\right\rangle$has an increasing trend with beam energy, whereas a horn structure is seen in the ratio of $\left\langle K^{+}\right\rangle /\left\langle\pi^{+}\right\rangle$. It has been shown 

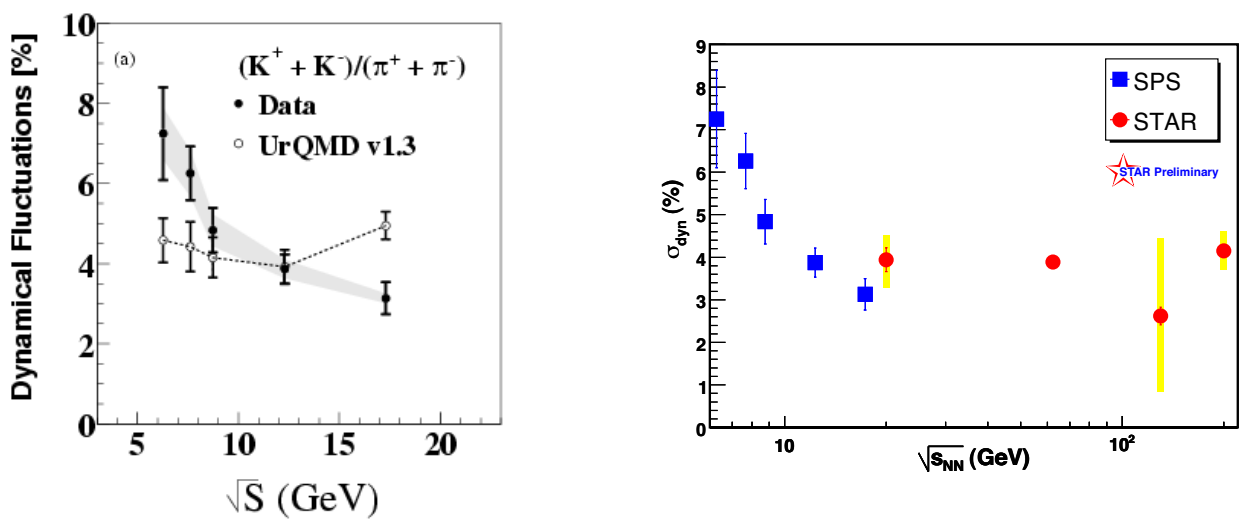

Figure 4. Excitation function for $\sigma_{d y n}$ of $\left[K^{+}+K^{-}\right] /\left[\pi^{+}+\pi^{-}\right]$ratio at the SPS (left panel) and with an extension to RHIC (right panel).

that the dynamical fluctuations $\left(\sigma_{\mathrm{dyn}}\right)$ in the ratio of $p / \pi$ has an increasing trend with respect to beam energy which could be explained by model calculations. At the same time $\sigma_{\mathrm{dyn}}$ in the $K / \pi$ ratio is seen to decrease with beam energy, a behavior which could not be explained by the same model. The $\sigma_{\text {dyn }}$ values at SPS energies are shown in the left panel of Fig. [4. The STAR experiment has performed a similar study on the event-wise fluctuations of the $K / \pi$ ratio for $\mathrm{Au}-\mathrm{Au}$ collisions at $\sqrt{s_{\mathrm{NN}}}=62.4 \mathrm{GeV}$ and $\sqrt{s_{\mathrm{NN}}}=200 \mathrm{GeV}$ [18. A reduction as a function of centrality is reported for the two energies. The right panel of Fig. 固 shows an excitation energy plot for $K / \pi$ ratio extended up to the highest RHIC energies. The fluctuation decreases with increasing energy up to the highest SPS energy and remains constant at higher RHIC energies. Theoretical investigations [19] are underway to explain such behaviour.

\section{5. $\left\langle p_{\mathrm{T}}\right\rangle$ fluctuations}

The $\left\langle p_{\mathrm{T}}\right\rangle$ of emitted particles in an event is related to the temperature of the system. Thus the event-by-event fluctuations of average $p_{\mathrm{T}}$ is sensitive to the temperature fluctuations predicted for the QGP phase transition. $\left\langle p_{\mathrm{T}}\right\rangle$ can be measured experimentally with high precision. The interpretation of the the results has to include considerations of acceptance effects, volume fluctuations, resonance decays, elliptic flow, HBT correlations, hard scattering and jet production. Several measures of fluctuation have been introduced in order to probe the dynamical fluctuation from the measured values. Figure 5 shows the centrality dependence of dynamical fluctuations reported by CERES [20], NA49 [21], PHENIX [22] and STAR [23]. The results presented in Fig. 5(d) show a smooth variation of fluctuation with centrality whereas the other measurements show non-monotonic behaviour. Efforts are being made to understand the nature and origin of these fluctuations. Because of the choice of several variables, extraction of an excitation energy plot combining data from SPS to RHIC is not straightforward. It is of interest to us to have a common framework for presenting the results from different 

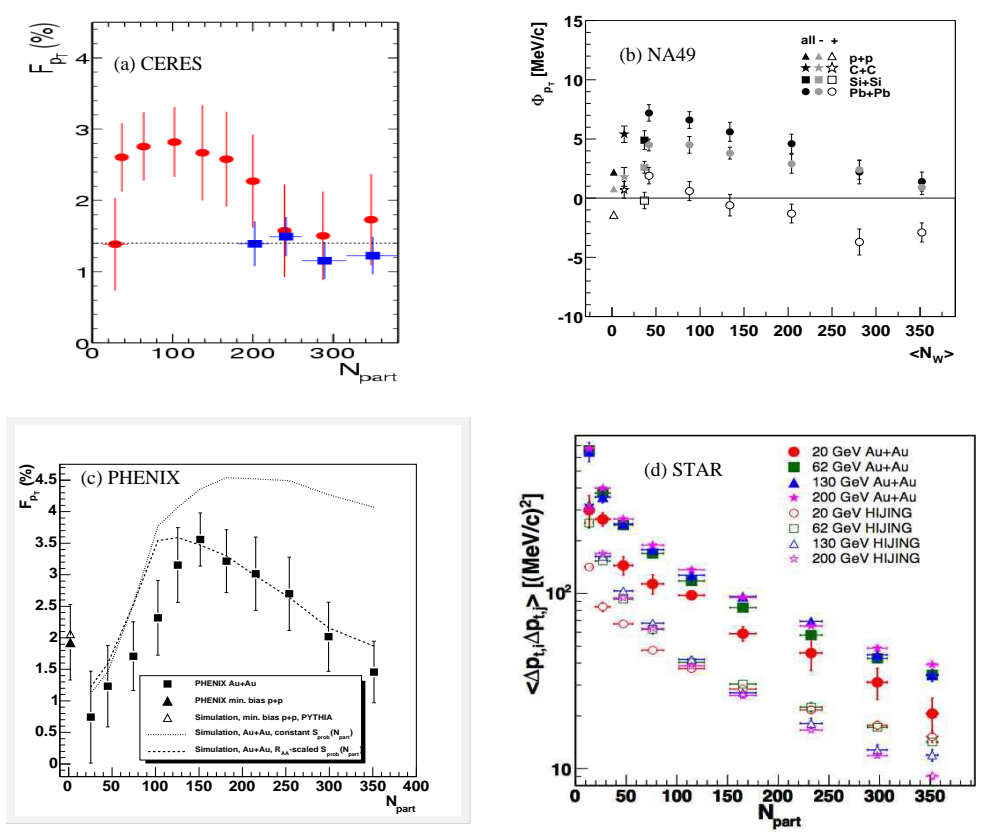

Figure 5. Dynamical $\left\langle p_{\mathrm{T}}\right\rangle$ fluctuations as a function of centrality of the collision from (a) CERES, (b) NA49, (c) PHENIX and (d) STAR experiments.
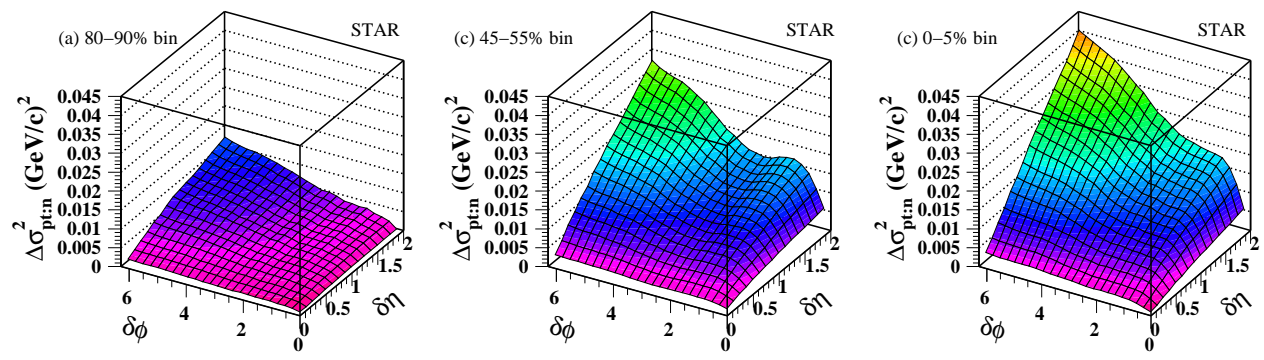

Figure 6. Scale dependence of $\left\langle p_{\mathrm{T}}\right\rangle$ fluctuation within the STAR acceptance expressed in terms of per-particle variance difference as discussed in [24].

experiments. In order to be more sensitive to the origin of fluctuations, differential measures have been adopted where the analysis is performed at different scales (varying bins in $\eta$ and $\phi$ ). The scale dependence of $\left\langle p_{\mathrm{T}}\right\rangle$ fluctuation for three centralities in $\mathrm{Au}-\mathrm{Au}$ collisions at $\sqrt{s_{\mathrm{NN}}}=200 \mathrm{GeV}$ [24] is shown in Fig. 6. The extracted autocorrelations are seen to vary rapidly with collision centrality, suggesting that fragmentation is strongly modified by a dissipative medium in more central collisions relative to peripheral collisions. Further studies for different charge combinations will provide more detailed information.

\section{Balance functions}

The method of Balance Functions (BF) [25], provides a measure of correlation of oppositely charged particles produced during heavy-ion collisions. The basic idea is that the charged hadrons are produced locally as oppositely charged-particle pairs. 

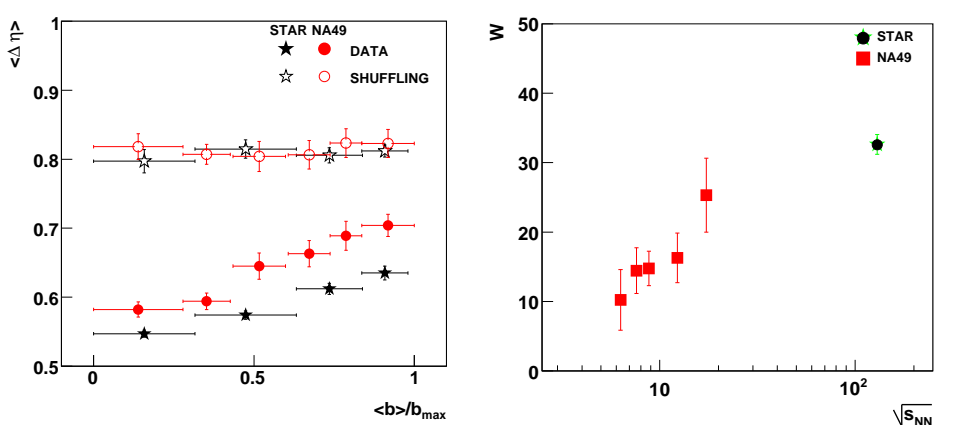

Figure 7. (a) The width of the balance function as a function of centrality for experimental data along with results for shuffled bins (b) Normalized parameter $(W)$ of balance function as a function of beam energy.

The particles of such a pair are separated in rapidity due to the initial momentum difference and secondary interaction with other particles. The particles of a pair produced earlier are separated further in rapidity compared to the particles coming from a pair produced later in time. Since the width of the correlation can be related to the time of hadronization of the charged particles, this would signal any possible delayed hadronization, corresponding to the formation of a high density QGP matter.

Both STAR [26] and NA49 [27] experiments have made detailed measurements of the BFs for various colliding systems, centralities, pseudorapidity intervals as well as for identified charged particles. Here we report two of these studies; centrality dependence and excitation energy dependence of BF widths. The left panel of Fig. 7 shows the width of the BFs as function of the normalized impact parameter for $\mathrm{Pb}-\mathrm{Pb}$ collisions at $\sqrt{s_{\mathrm{NN}}}=17.2 \mathrm{GeV}$ and $\mathrm{Au}-\mathrm{Au}$ collisions at $\sqrt{s_{\mathrm{NN}}}=130 \mathrm{GeV}$. The widths of the $\mathrm{BF}$ decrease from peripheral to central collisions in experimental data whereas the shuffled data shows no such reduction. The decrease in the width can be quantified by the use of a normalized parameter, $W$, expressed as enhancement in the width in the data with respect to the corresponding shuffled values. The values of $W$ are plotted in the right panel of Fig. 7 as a function of beam energy [28]. The increase of the $W$ from SPS to RHIC may be interpreted in terms of a delayed hadron scenario.

\section{Summary and outlook}

Experiments at SPS and RHIC have given a wealth of data on fluctuations of various observables, some of the interesting ones have been discussed here. The extraction of dynamical fluctuations originating from QGP phase transition from the experimental results becomes complicated because of several competing processes. This has been addressed from the available data on particle multiplicity, net charge, strangeness, and $\left\langle p_{\mathrm{T}}\right\rangle$. Differential measures are being adopted in order to gain insight to the details of fluctuation. Analysis based on forward-backward long range multiplicity correlations have recently been performed [29, 30] which show the presence of significant correlations 
for central collisions.

One of the most important aspects of QGP study is the location of the critical point. It may be possible to access this experimentally by scanning the QCD phase diagram in terms of baryon chemical potential and temperature. This can be accomplished

by varying beam energies from about $\sqrt{s_{\mathrm{NN}}}=5 \mathrm{GeV}$ to $100 \mathrm{GeV}$. Such a program is recently being undertaken at RHIC [31. Experiments at GSI 32 are planned to study this as well. At higher energies of LHC (Pb-Pb beams at $\left.\sqrt{s_{\mathrm{NN}}}=5.5 \mathrm{TeV}\right)$, the ALICE experiment will be able to make precise event-by-event measurements of various quantities and study their fluctuations 33. With continued development in new analysis methods and theoretical advances, and with dedicated experiments, one will certainly learn a great deal more about QGP phase transition through fluctuation studies.

\section{References}

[1] H. Heiselberg, Physics Reports 351 (2001) 161.

[2] M. A. Stephanov, K. Rajagopal and E. Shuryak, Phys. Rev. Lett. 81 (1998) 4816.

[3] M.M. Aggarwal et al., (WA98 Collaboration), Phys. Rev. C65, (2002) 054912.

[4] M. Rybczynski et al. (NA49 Collaboration), J. Phys. Conf. Ser. 4, (2005) 74.

[5] J. Mitchell et al. (PHENIX Collaboration), Preprint nucl-ex/0510076.

[6] M. Asakawa, U. Heinz, B.Muller, Phys. Rev. Lett. 85 (2000) 2072.

[7] S. Jeon and V. Koch, Phys. Rev. Lett. 85 (2000) 2076.

[8] E. Shuryak and M.A. Stephanov, Phys. Rev. C63 (2001) 064903.

[9] B. Mohanty, J. Alam, T.K. Nayak, Phys. Rev. C67 (2003) 024904.

[10] C. Alt et al. (NA49 Collaboration), Phys. Rev. C 70 (2004) 064903.

[11] J. Adams et al. (STAR Collaboration), Phys. Rev. C68 (2003) 044905.

[12] K. Adcox et al. (PHENIX Collaboration), Phys. Rev. Lett. 89 (2002) 082301.

[13] C. Pruneau, S. Gavin, S. Voloshin, Phys. Rev. C66 (2002) 044904.

[14] J.T. Mitchell, J. Phys G30 (2004) S819.

[15] S. Ejiri, F. Karsch and K. Redlich, Phys. Lett. B633 (2006) 275.

[16] R. V. Gavai and S. Gupta, Phys. Rev. D72 (2005) 054006.

[17] C. Roland et al. (NA49 Collaboration), J. Phys. G30 (2004) S1381.

[18] S. Das et al. (STAR Collaboration), Preprint nucl-ex/0503023.

[19] G. Torrieri, S. Jeon and J. Rafelski, Preprint nucl-th/0510024

[20] Hiroyuki Sato et al. (CERES Collaboration), J. Phys. G30 (2004) S1371.

[21] T. Anticic et al. (NA49 Collaboration), Phys. Rev. C70 (2004) 034902

[22] S.S. Adler et al. (PHENIX Collaboration), Phys. Rev. Lett. 93 (2004) 092301.

[23] J. Adams et al. (STAR Collaboration) Phys. Rev. C72 (2005) 044902.

[24] J. Adams et al. (STAR Collaboration) J. Phys. G32 (2006) L37.

[25] S. A. Bass, P. Danielewicz and S. Pratt, Phys. Rev. Lett. 85 (2000) 2689.

[26] J. Adams et al., (STAR Collaboration) Phys. Rev. Lett. 90 (2003) 172301.

[27] C. Alt et al. (NA49 Collaboration), Phys. Rev. C71 (2005) 034903;

[28] P. Christakoglou et al. (NA49 Collaboration), Preprint nucl-ex/0510045

[29] B.B. Back et al. (PHOBOS Collaboration) Phys. Rev. C74 (2006) 011901.

[30] Terence Tarnowsky et al. (STAR Collaboration), Preprint nucl-ex/0606018

[31] T. Ludlum et al. BNL-75692-2006, Proceedings of the workshop on "Can we discover the QCD Critical point at RHIC?", March 9-10, 2006.

[32] C. Hohne et al. (CBM COllaboration) Nucl. Phys. A749 (2005) 141.

[33] Physics Performance Report, Volume II, ALICE Collaboration, to be published in J. Phys. G. 